\title{
Manejo de florestas naturais degradadas na Amazônia: estudo de caso sobre critérios de colheita
}

\author{
Management of degraded natural forests in the Amazon: case study on harvest \\ criteria
}

\author{
Marco Antonio Siviero', Ademir Roberto Ruschel' ${ }^{\mathrm{II}}$, Jorge Alberto Gazel Yared ${ }^{\mathrm{III}}$, \\ Sabrina Benmuyal Vieira ${ }^{\mathrm{IV}}$, Agust Sales ${ }^{\mathrm{V}}$, José Francisco Pereira ${ }^{\mathrm{VI}}$, \\ Osmar José Romeiro de Aguiar ${ }^{\mathrm{VII}}$, Sílvio Brienza Junior ${ }^{\mathrm{VIII}}$, \\ Paulo Cezar Gomes Pereira ${ }^{\mathrm{IX}}$, Ghaby Alves Berberian ${ }^{\mathrm{X}}$, \\ Karina Piekarski Siviero Contini ${ }^{\mathrm{XI}}$
}

\begin{abstract}
Resumo
Este trabalho trata de uma experiência inovadora em relação a critérios para colheita de árvores em floresta natural degradada na Amazônia. A pesquisa foi desenvolvida em 535,6 ha de floresta na Fazenda Shet, em Dom Eliseu - PA, usando a base de dados do censo florestal referente a árvores com DAP $\geq$ $25 \mathrm{~cm}$. Os critérios para colheita das árvores consideraram, prioritariamente, os seguintes elementos: grau de sanidade, qualidade do fuste (2 e 3), diâmetro máximo a permanecer na floresta (DAP $<100 \mathrm{~cm}$ ), árvores de menor diâmetro $(25 \geq \mathrm{DAP} \leq 55 \mathrm{~cm})$, espécies com maior densidade de árvores por unidade de área, distribuição diamétrica, segundo o coeficiente de Liocourt, manutenção das espécies intensamente exploradas no passado e com população de árvores $\leq 0,15$ arv.ha ${ }^{-1}$. Foram inventariadas 46.012 árvores, pertencentes a 106 espécies, e planejadas para colheita 23,19\% (10.671 árvores), aos 12 anos após a exploração anterior. Baseando-se no planejamento da colheita e seguindo os critérios, a previsão de colheita em relação à população total inventariada teve como resultado: $2,16 \%$ árvores pelo critério de sanidade; $15,45 \%$ pela forma de fuste; $0,26 \%$ pelo diâmetro máximo; $93,93 \%$ pelo menor diâmetro; $57,50 \%$ pela densidade arbórea; e 5,04\% pela manutenção das espécies. A colheita foi realizada em $98,79 \%$ das árvores com sanidade comprometida; $22,20 \%$ com fuste 2 e 3; $97,39 \%$ com diâmetro máximo; $95,02 \%$ com menor diâmetro; e 90,30\% com maior densidade arbórea. Foram mantidas 98,14\% das espécies Astronium lecointei, Cordia goeldiana, Copaifera sp., Hymenaea courbaril, Hymenolobium petraeum, Handroanthus serratifolius e Manilkara elata, intensivamente exploradas no passado, e $98,70 \%$ de outras 53 espécies com menor abundância ( $\leq$ 0,15 arv.ha $^{-1}$ ). O planejamento da exploração seguindo os critérios de colheita propostos possibilitou a extração de árvores em ciclos de 10 a 12 anos, sendo um tempo menor que o previsto pela legislação. A manutenção da diversidade de espécies arbóreas e a conservação da floresta em pé, previstas com esses critérios técnicos, podem ser alternativas para o manejo florestal ecológica e economicamente viável.
\end{abstract}

Palavras-chave: Colheita florestal; Diâmetro de colheita; Colheita por distribuição de diâmetros; Manejo florestal

\footnotetext{
Engenheiro Mecânico, Economista, Diretor-Presidente do Grupo Arboris, Rua Gonçalves Dias, 919, Centro, CEP 6833-000, Dom Eliseu (PA), Brasil. m.g@masiviero.com.br (ORCID: 0000-0002-7232-3066)

Engenheiro Agrônomo, Dr., Embrapa Amazônia Oriental, Tv. Dr. Enéas Pinheiro, s/n, Marco, CEP 66095-903, Belém (PA), Brasil. ademir.ruschel@ embrapa.br (ORCID: 0000-0002-0352-5238)

III Engenheiro Florestal, Dr., Pesquisador-Colaborador do Grupo Arboris, Rua Gonçalves Dias, 919, Centro, CEP 6833-000, Dom Eliseu (PA), Brasil. jagyared@gmail.com (ORCID: 0000-0002-2595-755X)

IV Engenheira Florestal, Msc., Coordenadora de Pesquisa e Inovação do Grupo Arboris, Rua Gonçalves Dias, 919, Centro, CEP 6833-000, Dom Eliseu (PA), Brasil. sabrina.benmuyal@grupoarboris.com.br (ORCID: 0000-0001-8377-9280)

Engenheiro Florestal, MSc., Doutorando em Ciência Florestal, Universidade Federal de Viçosa, Av. Peter Henry Rolfs, s/n, Campus Universitário, CEP 36570-900, Viçosa (MG), Brasil. agustsales@hotmail.com (ORCID: 0000-0002-1364-9840)

VI Engenheiro Florestal, MSc., Embrapa Amapá, Rodovia Juscelino Kubitschek, Km 5, 2600, Universidade, CEP 68903-419, Macapá (AP). josefrancisco.pereira@embrapa.br (ORCID: 0000-0002-9807-2306)

vII Engenheiro Florestal, Dr., Professor do Centro de Ciências Tecnológicas, Universidade do Estado do Pará, Tv. Dr. Enéas Pinheiro, s/n, Marco, CEP 66095-903, Belém (PA), Brasil. o.aguiarromeiro@gmail.com (ORCID: 0000-0002-4417-6505)

vIII Engenheiro Florestal, Dr., Embrapa Amazônia Oriental, Tv. Dr. Enéas Pinheiro, s/n, Marco, CEP 66095-903, Belém (PA), Brasil. silvio.brienza@ embrapa.br (ORCID: 0000-0002-9313-7705)

IX Engenheiro Florestal, MSc., Professor da Universidade Federal Rural da Amazônia, PA-256, s/n, Nova Conquista, CEP 68625-970, Paragominas (PA), Brasil. paulo.pereira@ufra.edu.br (ORCID: 0000-0003-3317-4819)

Engenheira Florestal, Secretaria Municipal de Meio Ambiente de Dom Eliseu, Rua Gonçalves Dias, s/n, Centro, CEP 6833-000, Dom Eliseu (PA), Brasil. berberianghaby@yahoo.com.br (ORCID: 0000-0002-0364-5387)

XI Engenheira de Controle e Automação Industrial, Grupo Arboris - ADECO Indústria e Comércio de Compensados Ltda., BR010, km 16,5, Primavera, CEP 6833-000, Dom Eliseu (PA), Brasil. m.k@masiviero.com.br (ORCID: 0000-0002-9631-8632)
} 


\begin{abstract}
This paper deals with an innovative experience regarding tree harvesting criteria in degraded natural forest in the Amazon. The research was carried out in 535.6 ha of forest at Fazenda Shet, in Dom Eliseu/PA state, using the database of forest census for trees with $\mathrm{DBH} \geq 25 \mathrm{~cm}$. Criteria for tree harvesting considered, as a priority, the following elements: degree of health, shape of stem (2 and 3), maximum diameter to remain in the forest ( $\mathrm{DBH}=100$ $\mathrm{cm})$, smaller diameter trees $(25 \geq \mathrm{DBH} \leq 55 \mathrm{~cm})$, species with higher tree density per unit area, diameter distribution according to the Liocourt coefficient, maintenance of intensively exploited species in the past and tree population $\leq 0.15$ arv.ha $^{-1}$. A total of 46,012 trees belonging to 106 species were inventoried and planned for harvest $23.19 \%$ $(10,671$ trees) at 12 years after the previous harvest. Based on harvest planning and following the criteria, the harvest forecast in relation to the total inventoried population resulted in: $2.16 \%$ trees by the criterion of health; $15.45 \%$ for the shape of stem; $0.26 \%$ by the maximum diameter; $93.93 \%$ for the smallest diameter; $57.50 \%$ for the tree density; and $5.04 \%$ for the maintenance of the species. Harvesting was performed on $98.79 \%$ of trees with compromised health; $22.20 \%$ with stem 2 and 3; $97.39 \%$ with maximum diameter; $95.02 \%$ with smaller diameter; and $90.30 \%$ with higher tree density. Were kept $98.14 \%$ of the species Astronium lecointei, Cordia goeldiana, Copaifera sp., Hymenaea courbaril, Hymenolobium petraeum, Handrohanthus serratifolius and Manilkara elata, intensively exploited in the past, and $98.70 \%$ of 53 other species with less abundance $\left(\leq 0.15\right.$ arv.ha $\left.^{-1}\right)$. The exploration planning following the proposed harvesting criteria allowed the extraction of trees in cycles of 10 to 12 years, being a shorter time than the one foreseen by the legislation. The maintenance of tree species diversity and forest conservation, provided for with these technical criteria, may be alternatives to ecologically and economically viable forest management.
\end{abstract}

Keywords: Forest harvesting; Harvest diameter; Harvesting by diameter distribution; Forest management

\title{
Introdução
}

A compreensão dos serviços globais prestados pelas florestas fez com que a humanidade começasse a se preocupar com a conservação dos recursos florestais, iniciando-se um processo de formulação de medidas para proteger essa fonte de bens renováveis. No Brasil, várias iniciativas foram instituídas a fim de se preservar a floresta, especialmente por meio do Código Florestal e outras normas. Para a Amazônia, algumas dessas medidas foram: a) a criação da RL (Reserva Legal), com vistas à manutenção de $50 \%$ da área coberta com vegetação nativa na propriedade (BRASIL, 1965) e, posteriormente, 80\% (BRASIL, 2012); b) o estabelecimento do DMC (Diâmetro Mínimo de Corte) $\geq 50 \mathrm{~cm}$ de DAP (diâmetro a $1,30 \mathrm{~m}$ do solo) como critério para exploração das árvores no manejo florestal; c) o ciclo de corte de 25 a 35 anos para o período entre duas explorações (BRASIL, 2006; 2009; 2015) e; d) a Lei de Crimes Ambientais, Lei no 9.605/1998 (BRASIL, 1998), iniciando a prática do uso de comando e controle para coibir a atividade florestal ilegal.

$\mathrm{Na}$ última década, foi dada grande ênfase à política ambiental para coibir a ilegalidade de madeira. Nesse âmbito, ganhos ambientais importantes foram alcançados ainda que, muitas vezes, com medidas limitadoras e restritivas, colocando em risco a atividade florestal madeireira. $\mathrm{Na}$ mesma dimensão, deveriam ter ocorrido estímulos ou incentivos ao desenvolvimento da indústria de base florestal nativa, que passou a participar marginalmente na geração de emprego e renda nos estados da Amazônia. Esse fato reduziu expressivamente a participação do setor florestal na economia, com perda de $57 \%$ no consumo anual de toras, $55 \%$ em produção anual processada e 53\% em empregos gerados direta e indiretamente (IMAZON, 2005; 2010).

A existência de um enorme potencial das florestas primárias, sejam aquelas intensivamente exploradas no passado, sejam as florestas em reservas federais, estaduais ou privadas, clama pela concepção de políticas e legislação compatíveis com cada característica e tipologia florestal, de modo a valorizar esse recurso natural e fortalecer a indústria madeireira de base florestal. Com a adoção de um manejo florestal tecnicamente correto e baseado na vocação histórica da indústria madeireira, esse setor pode contribuir significativamente para o desenvolvimento sustentável da Amazônia.

A floresta adequadamente manejada pode ser uma fonte perene de suprimento para atender às necessidades humanas (VIEIRA et al., 2014). Os recursos florestais, em especial a 
madeira, são bens renováveis e, por isso, podem ser percebidos como ativo financeiro para o desenvolvimento econômico. Nesse sentido, as medidas legais devem auxiliar e fortalecer o uso racional da floresta por meio do manejo sustentável, com base em resultados de pesquisa relatados por Schwartz et al. (2017) e Sales et al. (2018), entre outros, posto que o manejo das florestas nativas é uma das principais alternativas de uso da terra para mantê-las em pé.

O objetivo deste estudo é apresentar uma experiência inovadora no planejamento da exploração florestal, com base na definição de critérios para colheita de árvores, em área de floresta natural intensivamente explorada no passado, visando manter a floresta em pé, garantir a produção florestal contínua e torná-la um ativo financeiro.

\section{Materiais e métodos}

\section{Caracterização e localização da área de estudo}

A área de estudo é uma floresta natural intensamente explorada, assim classificada como floresta degradada da região do arco do desflorestamento (SIVIERO et al., 2011; FEITOSA, 2015). A denominação de floresta degradada neste trabalho é caracterizada por uma floresta residual após sucessivas explorações ocorridas no passado para a retirada de espécies madeireiras nobres principalmente de grandes diâmetros. Ferreira et al. (2015) colocam que "as florestas degradadas são áreas que nunca deixaram de ser floresta, ou seja, nunca sofreram corte raso, porém, foram alteradas por ações antrópicas, como fogo e exploração não planejada”. Nessa área ocorreram ciclos de exploração em 1980, 1993/1994 e 2013/2014, em que foi plantado o paricá (Schizolobium parahyba var. amazonicum) em 1994, após a exploração ocorrida em 1993/1994. O plantio buscava enriquecimento ou adensamento florestal por meio da semeadura direta de sementes do paricá em todas as clareiras (SCHWARTZ et al., 2017).

A área de manejo florestal localiza-se na Fazenda Shet (535,6 ha), propriedade do Grupo Arboris, na cidade de Dom Eliseu, sudeste do estado do Pará ( $4^{\circ} 30^{\prime} 48^{\prime \prime S}$ e $47^{\circ} 39^{\prime} 36^{\prime}$ "W) (Figura 1).

Figura 1 - Localização da Fazenda Shet, em Dom Eliseu, Pará: mapa do bioma Amazônia; estado do Pará; e área de manejo florestal com suas sete unidades de trabalho

Figure 1 - Location of Fazenda Shet in Dom Eliseu, Pará: Map of the Amazon biome; state of Pará; and area of forest management with its seven work units

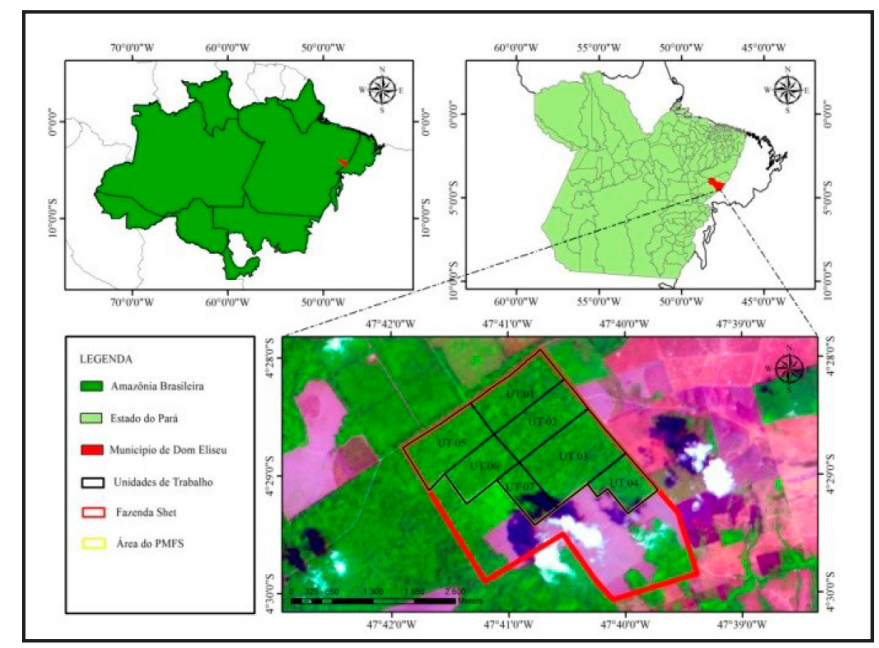

Fonte: Autores (2017) 
O clima do município é mesotérmico úmido. A temperatura média anual está em torno de $25^{\circ} \mathrm{C}$ e as médias das mínimas diárias, em cerca de $20^{\circ} \mathrm{C}$. Seu regime pluviométrico fica, normalmente, entre $2.250 \mathrm{~mm}$ e $2.500 \mathrm{~mm}$ ao ano. Apresenta variação em seus níveis altimétricos, cuja cota mínima está próxima de 76 metros, situada na porção noroeste do município, e a máxima em torno de $330 \mathrm{~m}$, localizada ao sul, na qual está a área de estudo (IDESP, 2014). No município predomina Floresta Ombrófila Densa Submontana Dossel Emergente, normalmente composta por árvores altas e relacionada a um clima quente e úmido (IBGE, 2012).

\section{Planejamento pré-colheita}

A área experimental foi dividida em sete unidades de trabalho (UT), separadas por estradas distantes a cada $300 \mathrm{~m}$, que compõem a infraestrutura permanente da área de manejo. Cada UT apresenta um número de pátios de estocagem proporcional a sua área, mantendo a relação de 1 para 30 ha. A dimensão de cada pátio foi definida em $20 \mathrm{~m}$ x $25 \mathrm{~m}$, alocados sistematicamente a cada $500 \mathrm{~m}$ na lateral da estrada secundária. Os pátios, instalados na primeira colheita florestal, foram planejados para serem usados nos próximos ciclos. A partir dos pátios foram direcionadas as trilhas de arraste.

\section{Censo florestal}

O censo florestal para este estudo foi realizado em 2008 seguindo a metodologia tradicional de inventário, conforme proposto por Sabogal et al. (2009). Na mesma ocasião, ocorreu a atividade de corte de cipós mormente nas árvores inventariadas. No inventário foram incluídas todas as árvores com DAP $\geq 25 \mathrm{~cm}$. Para cada árvore foram feitos os seguintes procedimentos: 1) identificação do nome vulgar por parabotânicos da região; 2) atribuição de numeração consecutiva com etiqueta de alumínio fixada na base do tronco; 3) medição do diâmetro a $1,30 \mathrm{~m}$ de altura e marcação com tinta vermelha no ponto de medição; 4) estimativa visual da altura comercial; 5) classificação quanto à forma de fuste (fuste-1: fuste reto, cilíndrico; fuste-2: levemente tortuoso e/ou canelado; fuste-3: fuste torto, fortemente canelado ou bifurcado); 6) observação do estado de sanidade (podridão, senescência, copa quebrada, morta, caída); e 7) registro das coordenadas cartesianas de localização (x,y).

No inventário algumas espécies foram agrupadas sob um mesmo nome vulgar, pela dificuldade de identificação naquela ocasião, como: amarelinho (Neoraputia paraensis, Metrodorea flavida, Amphiodon effusus); breu-amescla (Protium spp., Trattinnickia spp. e Tetragastris spp.); embaúba (Cecropia obtusa, Cecropia palmata, Cecropia sciadophylla, Cecropia distachya, Pourouma guianensis); freijó-branco (Cordia alliodora, Cordia bicolor, Cordia lomatoloba, Cordia caprifolia); ingá (Inga spp., Inga estipulares, Inga alba, Inga heterophylla); limãozinho (Zanthoxylum rhoifolia, Zanthoxylum ekimanii), matamatá (Eschweilera spp., Eschweilera ovata, Eschweilera coriacea, Eschweilera grandiflora, Lecythis idatimon); e tuturubá (Pouteria guianensis, Pouteria spp., Micropholis spp., Ecclinusa spp., Chrysophyllum spp.).

\section{Planejamento para a colheita}

No planejamento para a colheita, consideraram-se as árvores com DAP $\geq 25 \mathrm{~cm}$, para todas as espécies florestais, a partir dos critérios apresentados pelo Plano de Manejo Florestal Sustentável e pelo Plano Operacional Anual, por meio dos quais foi obtida a Autorização para a Exploração Florestal em 2013/2014 junto à Secretaria de Meio Ambiente e Sustentabilidade do Pará (SEMAS-PA) (PARA, 2014). Os critérios para a colheita estabelecidos no planejamento levaram em consideração, conjunta ou isoladamente, a classificação das condições silviculturais das árvores observadas em campo, conforme as seguintes especificações: 
Sanidade das árvores: deu-se prioridade à colheita das árvores passíveis de aproveitamento que apresentaram podridão, senescência, copa quebrada, sinais de doenças ou que estavam mortas, para evitar a perda de árvores por mortalidade e a propagação de insetos ou pragas nas árvores remanescentes. Tradicionalmente, o critério de sanidade das árvores não tem tido relevância na exploração convencional. No entanto, influi diretamente na qualidade da floresta futura.

Forma do fuste: priorizou-se a colheita de árvores com qualidade de fuste 2 e 3 e conservaram-se as árvores de fuste 1 (melhor qualidade de fuste);

Diâmetro máximo $(D A P=100 \mathrm{~cm})$ : estabeleceu-se que o diâmetro máximo desejado para árvores a permanecer na floresta seria DAP $=100 \mathrm{~cm}$. Esse critério de colheita foi adotado para reduzir a competição entre as árvores que ocupam o dossel, diminuir a população supostamente senescente e ajustar os diâmetros de árvores disponíveis ao processo industrial. A tendência é uma floresta nativa futura de produção em permanente crescimento, com maior número de árvores nas classes de diâmetros menores. A colheita de árvores de grande diâmetro no presente estudo visa compatibilizar a planta industrial futura adequada a um maior número de árvores de menor porte. Embora as árvores de maior diâmetro causem danos/aberturas na floresta, no processo de colheita se procurou administrá-las para minimizar a formação de grandes clareiras e infestação posterior de lianas (cipós).

Diâmetro menor para colheita $(25 \mathrm{~cm} \geq D A P \leq 50 \mathrm{~cm})$ : a colheita de árvores com diâmetros abaixo do previsto na legislação florestal (DAP $\leq 50 \mathrm{~cm}$ ) foi feita com objetivo de incluir no processo de produção árvores de espécies que por suas características biológicas não atingem o diâmetro de corte previsto nas normas e equilibrar a distribuição contínua das árvores em todas as classes diamétricas conforme o quociente de Liocourt.

População com altadensidade (arv.ha-1 ${ }^{-1}$ : definiu-se que a colheita deveria ocorrer preferencialmente para aquelas espécies com maior densidade arbórea.

Distribuição diamétrica: para aplicação do método BDq, utilizou-se o diâmetro máximo desejado de $100 \mathrm{~cm}$, como descrito anteriormente, e os valores de área basal remanescente (B) e quociente "q" de Liocourt (Tabela 1). Ajustou-se a frequência das classes de diâmetro, conforme o excedente das árvores, para todas as espécies em cada UT, segundo um quociente “q" de Liocourt (1898).

Tabela 1 - Valores de área basal remanescente, diâmetro máximo desejado e o quociente "q" de Liocourt planejados para cada UT, na Fazenda Shet, em Dom Eliseu, Pará

Table 1 - Values of remaining basal area, desired maximum diameter and Liocourt's "q" quotient planned for each Work Unit, in Fazenda Shet, in Dom Eliseu, Pará state

\begin{tabular}{lccccccc}
\hline \multicolumn{1}{c}{ UT } & $\mathbf{1}$ & $\mathbf{2}$ & $\mathbf{3}$ & $\mathbf{4}$ & $\mathbf{5}$ & $\mathbf{6}$ & $\mathbf{7}$ \\
\hline Área basal observada $\left(\mathbf{m}^{2} \cdot\right.$ ha $\left.^{-1}\right)$ & 11,48 & 12,17 & 9,61 & 6,31 & 11,98 & 10,14 & 4,82 \\
Área basal remanescente $\left(\mathbf{m}^{2} \cdot\right.$ ha $\left.^{-1}\right)$ & 9,00 & 9,00 & 7,00 & 4,70 & 9,50 & 7,00 & 4,00 \\
Quociente "q" & 2,30 & 2,30 & 2,30 & 2,70 & 2,40 & 2,30 & 2,60 \\
\hline
\end{tabular}

Fonte: Autores (2017) 
Prioridade para conservação das espécies: priorizou-se a manutenção das espécies Astronium lecointei (muiracatiara), Cordia goeldiana (freijó-cinza), Copaifera sp. (copaíba), Hymenaea courbaril (jatobá), Hymenolobium petraeum (angelim-pedra), Handroanthus serratifolius (ipê) e Manilkara elata (maçaranduba), que foram fortemente exploradas em colheitas anteriores. Essas espécies são conservadas como remanescente para favorecer a capacidade de reprodução e aumentar sua população na área.

População com baixa densidade arbórea $\left(\leq 0,15 \operatorname{arv} h a^{-1}\right)$ : estabeleceu-se a manutenção de um número mínimo de 15 árvores por espécie, com DAP $\geq 25 \mathrm{~cm}$, para cada 100 ha $(0,15$ arv.ha-1), para assegurar a perpetuação das populações de baixa densidade. As espécies classificadas por esse critério foram mantidas como matrizes para a produção de sementes e favorecimento da regeneração da espécie. As espécies estão sujeitas a características como umidade, iluminação, temperatura, que beneficiam ou não o seu desenvolvimento. Essas peculiaridades induzem as espécies desfavorecidas a ter um baixo número de árvores, as quais devem ser protegidas para que se tenha perpetuação da espécie e manutenção da biodiversidade.

\section{Processamento dos dados}

Os dados do Inventário Florestal a 100\% (IF 100\%) foram processados em planilha eletrônica Excel (Microsoft Office) de acordo com os critérios de sanidade, prioridade para conservação, população com baixa densidade, diâmetro máximo, diâmetro menor, forma de fuste, população com alta densidade e distribuição diamétrica pelo método BDq por UT.

\section{Resultados e discussão}

No censo florestal foram registradas 46.012 árvores, com DAP $\geq 25 \mathrm{~cm}$, pertencentes a 106 espécies. Da população inventariada planejou-se a colheita de $23,19 \%$ das árvores (10.671 árvores), concernentes a 53 espécies (Tabela 2; Material Suplementar 1). Da população inventariada, 2,16\% eram árvores com sanidade comprometida; $15,45 \%$ eram árvores com forma de fuste 2 e 3; 0,26\% árvores tinham diâmetro máximo e $93,93 \%$ árvores tinham menor diâmetro; $57,50 \%$ fazem parte de árvores pertencentes a espécies de maior densidade arbórea e 5,04\% pertenciam a árvores de espécies com prioridade para conservação (Tabelas 3, 4, 5, 6, 7, 8, respectivamente).

Neste estudo, a colheita foi realizada em $98,79 \%$ das árvores com sanidade comprometida; $22,20 \%$ com fuste 2 e 3; $97,39 \%$ com diâmetro máximo e 95,02\% com menor diâmetro; $90,30 \%$ com maior densidade arbórea, mantendo-se 98,14\% das espécies Astronium lecointei, Cordia goeldiana, Copaifera sp., Hymenaea courbaril, Hymenolobium petraeum, Handroanthus serratifolius e Manilkara elata; e 98,70\% de outras 53 espécies com menor abundância $\left(\leq 0,15\right.$ arv.ha-1 ${ }^{-1}$. Além desses critérios, foi ajustado o balanceamento da floresta pelo quociente "q" de Liocourt, que mostrou o excedente e deficit de árvores na distribuição diamétrica (Figura 3; Tabelas 3, 4, 5, 6, $7,8,9)$. 
Tabela 2 - Número de árvores inventariadas (DAP $\geq 25 \mathrm{~cm}$ ) e volume, destinadas para colheita e remanescente, nas sete UT, na Fazenda Shet, em Dom Eliseu, Pará

Table 2 - Number of trees and volume inventoried, for harvest and remaining, in the seven work units, in Shet Farm, in Dom Eliseu, Pará state

\begin{tabular}{|c|c|c|c|c|c|c|c|c|}
\hline UT & 1 & 2 & 3 & 4 & 5 & 6 & 7 & Total \\
\hline Área total (ha) & 82,70 & 83,70 & 129,60 & 50,50 & 88,20 & 59,30 & 41,60 & 535,60 \\
\hline N. absoluto & 7.805 & 8.443 & 10.241 & 3.348 & 8.829 & 5.499 & 1.847 & 46.012 \\
\hline N.ha-1 & 94,38 & 100,87 & 79,02 & 66,30 & 100,10 & 92,73 & 44,40 & 85,91 \\
\hline NPC & 1.681 & 2.083 & 2.275 & 830 & 1.679 & 1.814 & 309 & 10.671 \\
\hline NPC.ha-1 & 20,33 & 24,89 & 17,55 & 16,44 & 19,04 & 30,59 & 07,43 & 19,92 \\
\hline$\%$ NPC & 21,54 & 24,67 & 22,21 & 24,79 & 19,02 & 32,99 & 16,33 & 23,19 \\
\hline$V$ total $\left(\mathbf{m}^{3}\right)$ & $9.421,02$ & $9.668,98$ & $12.238,82$ & $2.884,81$ & $11.578,24$ & $6.087,18$ & $2.138,12$ & $54.017,16$ \\
\hline $\mathrm{V} / \mathbf{h a}\left(\mathbf{m}^{3} \cdot \mathbf{h a}^{-1}\right)$ & 113,92 & 115,52 & 94,44 & 57,13 & 131,27 & 102,65 & 51,40 & 100,85 \\
\hline $\operatorname{VPC}\left(\mathbf{m}^{3}\right)$ & $2.404,45$ & $3.056,24$ & $3.948,68$ & 973,98 & $2.649,20$ & $2.602,71$ & 432,28 & $16.067,53$ \\
\hline VPC /ha $\left(\mathbf{m}^{3} \cdot \mathbf{h a}^{-1}\right)$ & 29,07 & 36,51 & 30,47 & 19,29 & 30,04 & 43,89 & 10,39 & 30,00 \\
\hline$\%$ VPC & 25,52 & 31,60 & 32,26 & 33,76 & 22,88 & 42,76 & 20,22 & 29,74 \\
\hline
\end{tabular}

Fonte: Autores (2017)

Em que: N. - número de árvores; NPC - número de árvores para colheita absoluto e por hectare; V - volume absoluto e por hectare; VPC - volume para colheita absoluto e por hectare; UT - unidade de trabalho.

\section{Sanidade das árvores}

As árvores qualificadas pelo critério sanidade representam 2,16\% (993 árvores) do total inventariado e pertencem a 48 espécies. Desse total, 98,79\% (981 árvores) foram planejadas para colheita. As espécies que apresentaram maior nível de dano foram embaúba, ingá, breu-amescla, tuturubá e limãozinho. Essas espécies representaram 69,18\% (687 árvores) da população com problemas de sanidade (Tabela 3).

O problema de sanidade das árvores é uma das principais causas da mortalidade de árvores (FONTES, 2012; FONTES et al., 2018). Essa perda é avaliada prioritariamente quanto à importância da ciclagem de nutrientes, excluindo a hipótese de essas árvores serem precursoras de doenças que, biologicamente, podem ser perpetuadas em seus descendentes (POGGIANI, 1980). 
Tabela 3 - Espécies e número de árvores classificadas pelo critério de sanidade, no IF 100\%, na Área de Manejo da Fazenda Shet, em Dom Eliseu, Pará

Table 3 - Species and number of trees classified by the criterion of health, in $100 \%$ IF, in Fazenda Shet Management area, in Dom Eliseu, Pará state

\begin{tabular}{|c|c|c|c|c|c|c|}
\hline & Nome científico & Nome popular & 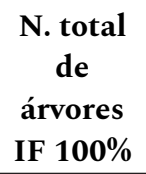 & $\begin{array}{c}\% \\
\text { população } \\
\text { doente }\end{array}$ & $\begin{array}{c}\text { N. árvores com } \\
\text { problemas de } \\
\text { sanidade }\end{array}$ & $\begin{array}{l}\% \text { planejado } \\
\text { para colheita } \\
\text { por sanidade }\end{array}$ \\
\hline 1 & Cecropia obtusa & Embaúba & 6.805 & 3,82 & 260 & 26,18 \\
\hline 2 & Inga spp. & Ingá & 2.729 & 6,67 & 182 & 18,32 \\
\hline 3 & Trattinnickia burseraefolia & Breu-amescla & 3.912 & 3,50 & 137 & 13,79 \\
\hline 4 & Pouteria guianensis & Tuturubá & 1.164 & 6,87 & 80 & 08,06 \\
\hline 5 & Zanthoxylum rhoifolium & Limãozinho & 1.224 & 2,29 & 28 & 02,81 \\
\hline \multicolumn{3}{|c|}{ Subtotal } & 15.834 & 4,34 & 687 & 69,18 \\
\hline \multicolumn{3}{|c|}{ Demais espécies (43 spp.) } & 30.178 & 1,01 & 306 & 30,82 \\
\hline \multicolumn{3}{|c|}{ Planejada colheita (48 spp.) } & 10.671 & 9,19 & 981 & 98,79 \\
\hline \multicolumn{3}{|c|}{ Total geral } & 46.012 & 2,16 & 993 & 100,00 \\
\hline
\end{tabular}

Fonte: Autores (2017)

Em que: N. - número de árvores; \% - percentual; IF - Inventário Florestal.

\section{Forma do fuste}

As árvores com fuste 2 e 3 corresponderam a 15,45\% da população total inventariada, distribuídos em 83 espécies. Desse total, 22,20\% (1.578 árvores) foram planejadas para colheita. No caso específico da Fazenda Shet, as espécies que representaram destacadamente a aplicação desse critério foram amarelinho, amescla, envira-quiabo, freijó-branco, ingá, limãozinho, pelede-sapo, quina e tuturubá. Dessas espécies, foram inventariadas 12.246 árvores, perfazendo $26,61 \%$ da população total, e planejadas para colheita $14,7 \%$ das árvores que apresentaram fuste $1 ; 23,8 \%$ do fuste 2 ; e $45,3 \%$ do fuste 3 (Tabela 4 ).

A análise do fuste foi realizada de acordo com a qualidade requerida para o processamento industrial. A maioria das espécies, como paricá, jatobá, maçaranduba, entre outras, apresenta fuste retilíneo com perímetro semelhante a um círculo, classificado como fuste 1 . No entanto, outras, como o barrote, apresentam dois outros casos de forma de fuste: fuste 2 , que é levemente tortuoso e/ou canelado; e fuste 3 , torto, fortemente canelado ou bifurcado. 
Tabela 4 - Número absoluto e percentagem das árvores de espécies representativas do critério forma de fuste, planejadas para colheita, no IF $100 \%$, na Área de Manejo Florestal da Fazenda Shet, em Dom Eliseu, Pará

Table 4 - Absolute number and percentage of the trees of representative species of the criterion shape of stem, planned for harvest, in the $100 \% \mathrm{IF}$, in the forest management area of Fazenda

Shet, in Dom Eliseu, Pará state

\begin{tabular}{|c|c|c|c|c|c|c|c|c|c|c|c|}
\hline & \multirow[b]{2}{*}{ Nome científico } & \multirow[b]{2}{*}{$\begin{array}{c}\text { Nome } \\
\text { popular }\end{array}$} & \multicolumn{3}{|c|}{ Fuste 1} & \multicolumn{3}{|c|}{ Fuste 2} & \multicolumn{3}{|c|}{ Fuste 3} \\
\hline & & & $\begin{array}{c}\mathrm{N} . \\
\text { total }\end{array}$ & $\begin{array}{c}\text { N. } \\
\text { colheita }\end{array}$ & $\%$ & $\begin{array}{c}\mathrm{N} . \\
\text { total }\end{array}$ & $\begin{array}{c}\text { N. } \\
\text { colheita }\end{array}$ & $\%$ & $\begin{array}{c}\mathrm{N} . \\
\text { total }\end{array}$ & $\begin{array}{c}\text { N. } \\
\text { colheita }\end{array}$ & $\%$ \\
\hline 1 & Amphiodon effusa & Amarelinho & 547 & 107 & 19,6 & 32 & 10 & 31,3 & 16 & 14 & 87,5 \\
\hline 2 & $\begin{array}{l}\text { Trattinnickia } \\
\text { burseraefolia }\end{array}$ & Amescla & 2.561 & 475 & 18,5 & 840 & 290 & 34,5 & 374 & 205 & 54,8 \\
\hline 3 & Sterculia pruriens & $\begin{array}{l}\text { Envira- } \\
\text { quiabo }\end{array}$ & 928 & 136 & 14,7 & 105 & 17 & 16,2 & 68 & 22 & 32,4 \\
\hline 4 & Cordia alliodora & $\begin{array}{l}\text { Freijó- } \\
\text { branco }\end{array}$ & 877 & 83 & 9,5 & 58 & 20 & 34,5 & 22 & 6 & 27,3 \\
\hline 5 & Inga sp. & Ingá & 1.640 & 239 & 14,6 & 727 & 148 & 20,4 & 180 & 96 & 53,3 \\
\hline 6 & $\begin{array}{l}\text { Zanthoxylum } \\
\text { rhoifolium }\end{array}$ & Limãozinho & 1.057 & 161 & 15,2 & 106 & 40 & 37,7 & 33 & 19 & 57,6 \\
\hline 7 & Pausandra trianae & $\begin{array}{l}\text { Pele-de- } \\
\text { sapo }\end{array}$ & 362 & 9 & 2,5 & 308 & 9 & 2,9 & 25 & 14 & 56,0 \\
\hline 8 & $\begin{array}{l}\text { Geissospermum } \\
\text { sericeum }\end{array}$ & Quina & 95 & 3 & 3,2 & 49 & 2 & 4,1 & 152 & 49 & 32,2 \\
\hline 9 & Pouteria guianensis & Tuturubá & 821 & 95 & 11,6 & 91 & 15 & 16,5 & 172 & 47 & 27,3 \\
\hline \multicolumn{2}{|c|}{ Total amostral } & & 8.888 & 1.308 & 14,7 & 2.316 & 551 & 23,8 & 1.042 & 472 & 45,3 \\
\hline \multicolumn{2}{|c|}{ Demais 31 espécies } & & 29.023 & 6.805 & 23,4 & 2.279 & 285 & 12,5 & 1.471 & 270 & 18,4 \\
\hline \multicolumn{2}{|c|}{ Total geral } & & 37.911 & 8.113 & 38,1 & 4.595 & 836 & 36,3 & 2.513 & 742 & 63,7 \\
\hline
\end{tabular}

Fonte: Autores (2017)

Em que: N. - número de árvores; \% - percentual; IF - Inventário Florestal.

\section{Diâmetro máximo $(\mathrm{DAP}=100 \mathrm{~cm})$}

A população com DAP superior a $100 \mathrm{~cm}$ foi de 119 árvores, correspondentes a $0,26 \%$ da população total inventariada. Desse grupo, foram planejadas para colheita 104 árvores, representando 97,39\% de 20 espécies (Tabela 5). Destacam-se caneleiro, angico, quina, amescla, taúba, goiabão, atraca, guajará e tuturubá. As 15 árvores restantes foram mantidas pelo critério de população com baixa densidade. 
Tabela 5 - Número de árvores inventariadas e planejadas para colheita nas classes de diâmetro máximo de colheita, no IF 100\%, na Área de Manejo Florestal da Fazenda Shet, em Dom Eliseu, Pará

Table 5 - Number of trees inventoried and planned for harvesting in classes of maximum harvest diameter, in the $100 \%$ IF, in the forest management area of Fazenda Shet, in Dom Eliseu, Pará

\begin{tabular}{lcc}
\hline Classe de DAP & N. inv. & N. planejado para colheita \\
\hline $\mathbf{1 0 5}-115$ & 51 & 46 \\
$\mathbf{1 1 5}-125$ & 39 & 35 \\
$\mathbf{1 2 5}-135$ & 10 & 9 \\
$\mathbf{1 3 5}-\mathbf{1 4 5}$ & 3 & 2 \\
$\mathbf{1 4 5}-\mathbf{1 5 5}$ & 8 & 5 \\
$\mathbf{1 5 5}-\mathbf{1 6 5}$ & 3 & 2 \\
$\mathbf{1 7 5}-\mathbf{1 8 5}$ & 1 & 1 \\
$\mathbf{1 8 5}-195$ & 3 & 3 \\
$\mathbf{1 9 5 - 2 0 5}$ & 1 & 1 \\
\hline Total & 119 & 104 \\
\hline
\end{tabular}

Fonte: Autores (2017)

Em que: N. inv. - número de árvores inventariadas; N. - número de árvores; IF Inventário Florestal.

\section{Diâmetro menor para colheita $(20 \mathrm{~cm} \geq \mathrm{DAP} \leq 55 \mathrm{~cm})$}

No censo florestal da Fazenda Shet o menor diâmetro para colheita foi de $25 \mathrm{~cm}$. Da população total inventariada, 93,93\% das árvores estavam presentes nas classes de diâmetro de 25 a $55 \mathrm{~cm}$, com 43.220 árvores, pertencentes a 104 espécies. Da população planejada para colheita, 95,02\% foram árvores com o diâmetro menor, distribuídas em 48 espécies, cuja dominância estava concentrada em paricá, embaúba, amescla, limãozinho, tuturubá e envira-quiabo (Tabela 6).

Tabela 6 - Número de árvores inventariadas e planejadas para colheita nas classes de diâmetros menores, no IF 100\%, na Área de Manejo Florestal da Fazenda Shet, em Dom Eliseu, Pará

Table 6 - Number of trees inventoried and planned for harvesting in the classes of smaller diameters, in the $100 \%$ IF, in the forest management area of Fazenda Shet, in Dom Eliseu, Pará state

\begin{tabular}{lccc}
\hline Classe de DAP & N. inv. & N. arv. planejado para colheita & \% planejada para colheita \\
\hline $\mathbf{2 5}-\mathbf{3 5}$ & 24.726 & 3.297 & 30,89 \\
$\mathbf{3 5 - 4 5}$ & 13.314 & 5.213 & 48,85 \\
$\mathbf{4 5 - 5 5}$ & 5.180 & 1.631 & 15,28 \\
Subtotal & 43.220 & 10.141 & 95,02 \\
Total & 46.012 & 10.672 & 100,00 \\
\hline
\end{tabular}

Fonte: Autores (2017)

Em que: DAP: N. inv. - número de árvores inventariadas; N. arv. - número de árvores; \% - percentual; IF - Inventário Florestal. 


\section{População com alta densidade (arv.ha $\left.{ }^{-1}\right)$}

As dez espécies com maior densidade arbórea corresponderam a 57,5\% da população inventariada e representaram $90,3 \%$ do total das árvores planejadas para colheita. Entre essas, destacam-se o paricá (plantado em clareiras), embaúba, amescla, ingá, limãozinho, tuturubá, embira-quiabo, freijó-branco, inharé e pitomba (Tabela 7).

Tabela 7 - Abundância das espécies conforme o planejamento para colheita do IF 100\%, na área de manejo florestal da Fazenda Shet, em Dom Eliseu, Pará

Table 7 - Abundance of the species according to the 100\% IF harvest planning, in the forest management area of the Fazenda Shet, in Dom Eliseu, Pará state

\begin{tabular}{|c|c|c|c|c|c|c|c|}
\hline N. & Nome científico & $\begin{array}{c}\text { Nome } \\
\text { popular }\end{array}$ & $\begin{array}{c}\mathrm{N} . \\
\text { total }\end{array}$ & $\begin{array}{c}\mathrm{N} . \\
\text { colheita }\end{array}$ & N. RM & $\begin{array}{c}\% \text { total } \\
\text { inventariado }\end{array}$ & $\begin{array}{c}\% \text { planejado } \\
\text { para colheita }\end{array}$ \\
\hline 1 & $\begin{array}{l}\text { Schizolobium parahyba } \\
\text { var. amazonicum }\end{array}$ & Paricá & 6.899 & 4.440 & 2.459 & 15,0 & 41,6 \\
\hline 2 & Cecropia obtusa & Embaúba & 6.805 & 2.443 & 4.362 & 14,8 & 22,9 \\
\hline 3 & Trattinnickia burseraefolia & Amescla & 3.912 & 1.107 & 2.805 & 08,5 & 10,4 \\
\hline 4 & Inga spp. & Ingá & 2.729 & 6.65 & 2.064 & 05,9 & 06,2 \\
\hline 5 & Zanthoxylum rhoifolium & Limãozinho & 1.224 & 2.48 & 976 & 02,7 & 02,3 \\
\hline 6 & Pouteria guianensis & Tuturubá & 1.164 & 237 & 927 & 02,5 & 02,2 \\
\hline 7 & Sterculia pruriens & $\begin{array}{l}\text { Envira- } \\
\text { quiabo }\end{array}$ & 1.110 & 184 & 926 & 02,4 & 01,7 \\
\hline 8 & Cecropia obtusa & $\begin{array}{l}\text { Freijó- } \\
\text { branco }\end{array}$ & 977 & 129 & 848 & 02,1 & 01,2 \\
\hline 9 & Helicostylis pedunculata & Inharé & 820 & 105 & 715 & 01,8 & 01,0 \\
\hline 10 & Talisia sp. & Pitomba & 819 & 75 & 744 & 01,8 & 09,2 \\
\hline \multicolumn{2}{|c|}{ Subtotal } & & 26.459 & 9.633 & 16.826 & 57,5 & 90,3 \\
\hline \multicolumn{2}{|c|}{ Total geral } & & 46.012 & 10.672 & 35.340 & 100,0 & 100,0 \\
\hline
\end{tabular}

Fonte: Autores (2017)

Em que: N. - número de árvores; RM - remanescente; \% - percentual; IF - Inventário Florestal.

O paricá e a embaúba apresentaram $29,8 \%$ de árvores do total inventariado. A elevada densidade do paricá é explicada pelo plantio de sementes; já a de embaúba é decorrente da regeneração natural favorecida pela alta quantidade de clareiras causadas pelas colheitas anteriores. A densidade arbórea das outras espécies (amescla, ingá, limãozinho, tuturubá, enviraquiabo, freijó, inharé e pitomba) pode ser justificada pelo agrupamento de várias espécies sob um mesmo nome popular (Tabela 7).

A atividade silvicultural de enriquecimento com paricá promoveu um ganho florestal médio nas sete UT de 40\% quanto ao número de árvores (Figura 2C) e 40,1\% quanto ao volume total planejado para colheita (Figura 2D). Tal prática de manejo silvicultural foi efetiva para aumentar o número de árvores dessa espécie na área, podendo ser uma técnica propícia para outras espécies que apresentam dificuldades de regeneração ou cuja abundância se pretenda 
aumentar (VATRAZ et al., 2012; VATRAZ et al., 2016; SCHWARTZ et al., 2017).

A elevada densidade natural da embaúba e sua introdução na cadeia de produção possibilitou um ganho médio de $25,1 \%$ quanto ao número de árvores (Figura 2C) e de 20,9\% quanto ao volume total planejado para colheita (Figura 2D). Neste caso, por ser uma espécie de ciclo de vida curto, as colheitas na floresta podem ocorrer em períodos inferiores a 35 anos, compatíveis com o ciclo de vida da espécie. A experiência sugere colheitas entre 10 e 12 anos, evitando, assim, a perda de madeira por mortalidade de árvores (SIVIERO, 2007; 2009; PEREIRA, 2015).

As clareiras adensadas/enriquecidas com paricá adicionada à regeneração natural de espécimes do gênero Cecropia, ambas pioneiras de ciclo de vida curto, introduzidas na cadeia de produção florestal, contribuíram conjuntamente com $65,1 \%$ das árvores colhidas (Figura 2C) e mais da metade do volume florestal colhido (61\%) (Figura 3D). Dessa forma, foram colhidas preferencialmente as populações com maior densidade.

As UT que expressaram maior densidade de paricá foram UT3, UT4 e UT6, resultante da atividade de enriquecimento. As UT com maior abundância de Cecropia foram UT1, UT7 e UT5, resultante da regeneração natural da espécie (Figura 2A). O paricá e as Cecropia fazem parte da cadeia de produção de painel de compensado na Adeco Indústria e Comércio de Compensados Ltda., em Dom Eliseu, Pará, e constituem-se como espécies importantes na recuperação da floresta (BRAGA et al., 2008; IWAKIRI et al., 2010; GARZÓN; PAOLA, 2016).

Figura 2 - Detalhes da distribuição percentual dos espécimes de embaúba, paricá $\mathrm{e}$ demais espécies da comunidade: A - árvores do Inventário Florestal 100\%; B - volume do Inventário Florestal $\mathbf{1 0 0} \%$; C - árvores selecionadas à colheita; e D - volume selecionado à colheita, em cada UT. Em que: UT - Unidade de Trabalho; IF - Inventário Florestal

Figure 2 - Details of distribution percentage of specimens of embaúba, paricá and other community species: A - trees of forest inventory $100 \%$; B - volume of forest inventory $100 \%$; C - trees selected for harvest and; D - volume selected for harvest, in each work unit. Where: UT

- Work Unit; IF - Forest Inventory

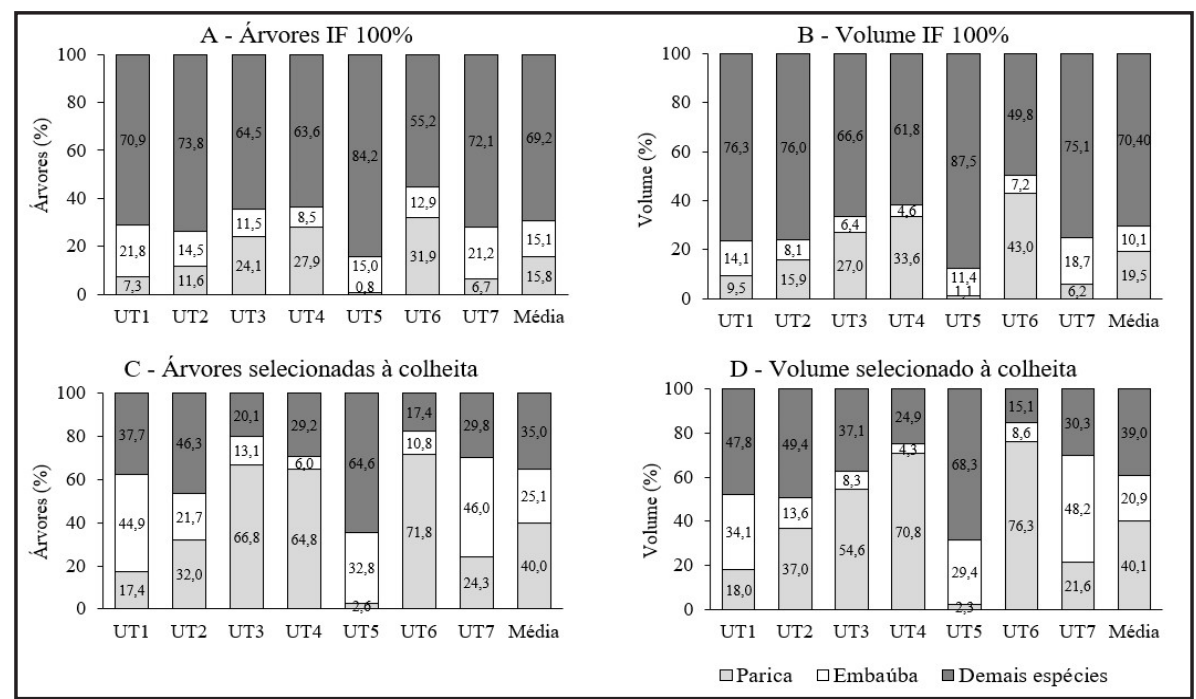

Fonte: Autores (2017)

\section{Distribuição diamétrica}

A aplicação do método BDq das sete UT é representado na Figura 3, em que podem ser 
observadas as classes diamétricas nas quais há superavit no número de árvores em relação à curva balanceada ajustada (curva remanescente) e classes diamétricas com deficit de árvores. Por meio do balanceamento, propôs-se a colheita de árvores nas classes superavitárias (curva colheita) até atingirem o número desejado remanescente e a manutenção das árvores nas classes deficitárias.

Figura 3 - Distribuição diamétrica das árvores observadas, remanescentes e para colheita, em cada UT da Fazenda Shet, em Dom Eliseu, Pará. Em que: NOB - número de árvores observadas; NREM - número de árvores remanescentes; NCUT - número de árvores colhidas; e UT - Unidade de trabalho

Figure 3 - Diametric distribution of the observed trees, remaining and harvesting in each work unit of Fazenda Shet Farm in Dom Eliseu, Pará state. Where: NOBS - number of trees observed; NREM - number of trees remaining; NCUT - number of trees to harvest; and UT Work Unit

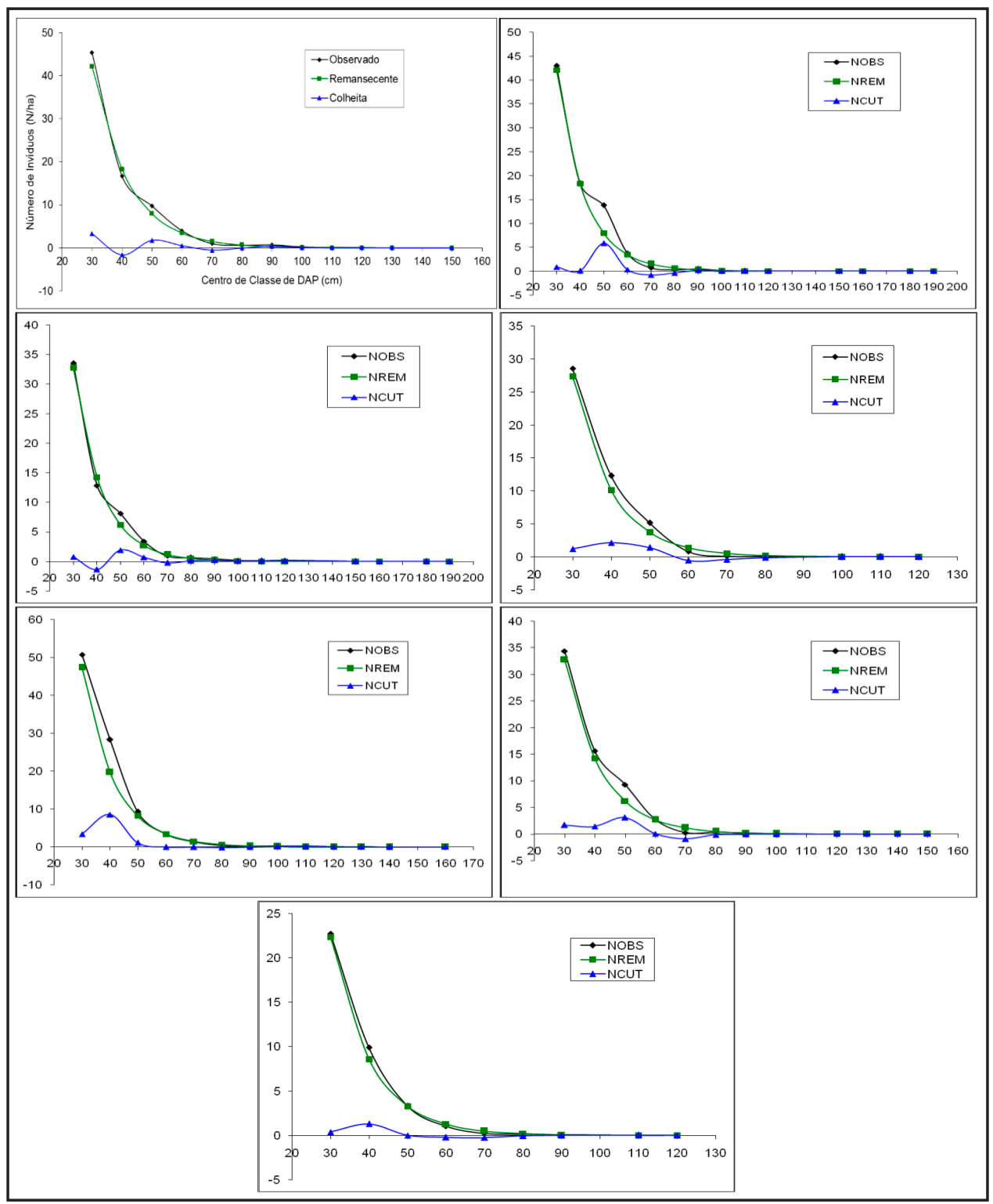

Fonte: Autores (2014) 
Nas florestas antropizadas é comum encontrar-se desequilíbrio, com abundância ou falta de árvores em determinada classe de diâmetro, refletindo o resultado da ação humana (LIOCOURT, 1898; MEYER, 1952; SOUZA, 2005). No entanto, observa-se que a floresta mantém sua estrutura na forma de J-invertido e apresenta, como mostrado em outros estudos, estoque de árvores suficientes para se recuperar (FIGUEIREDO FILHO et al., 2010; LIMA et al., 2013).

\section{Conservação das espécies}

Foram inventariadas 2.318 árvores de espécies fortemente exploradas em colheitas anteriores, perfazendo 5,04\% da população total. Desse montante, foram mantidas 2.275 árvores $(98,14 \%)$. As 43 árvores restantes foram destinadas à colheita por problemas de sanidade (Tabela $8)$.

Tabela 8 - Número de árvores inventariadas, árvores destinadas para colheita e remanescentes, das espécies que tiveram prioridade para conservação, na área de manejo florestal da Fazenda Shet, em Dom Eliseu, Pará

Table 8 - Number of trees planted for harvest and remnant trees of the species that were conserved in the forest management area of the Shet Farm in Dom Eliseu, Pará state

\begin{tabular}{llccccc}
\hline & Nome científico & Nome popular & N. inv. & \% inv. & Colheita & Remanescente \\
\hline $\mathbf{1}$ & Hymenolobium petraeum & Angelim-pedra & 139 & 0,30 & 0 & 139 \\
$\mathbf{2}$ & Cedrela odorata & Cedro & 57 & 0,12 & 0 & 57 \\
$\mathbf{3}$ & Copaifera spp. & Copaíba & 85 & 0,18 & 0 & 85 \\
$\mathbf{4}$ & Cordia goeldiana & Freijó & 574 & 1,25 & 15 & 559 \\
$\mathbf{5}$ & Handroanthus serratifolius & Ipê & 280 & 0,61 & 8 & 272 \\
$\mathbf{6}$ & Hymenaea courbaril & Jatobá & 636 & 1,38 & 6 & 630 \\
$\mathbf{7}$ & Manilkara elata & Maçaranduba & 345 & 0,75 & 14 & 331 \\
$\mathbf{8}$ & Astronium lecointei & Muiracatiara & 202 & 0,44 & 0 & 202 \\
\hline \multicolumn{2}{|}{ Total geral } & & 2.318 & 5,04 & $43^{\star}$ & 2.275 \\
\hline
\end{tabular}

Fonte: Autores (2017)

Em que: N. inv. - número de árvores inventariadas; \% inv. - percentual inventariado.

No critério conservação também se priorizou a manutenção de espécies com baixa densidade populacional $\left(\leq 0,15\right.$ arv.ha $\left.{ }^{-1}\right)$, das quais foram inventariadas 927 árvores de 53 espécies, representando $2,01 \%$ da população total inventariada, e mantiveram-se 915 árvores (98,70\%). Entre elas destacam-se: quina-rosa, casca-seca, sapucaia, jatobá-curuba, serigarana e amarelão, as quais não foram colhidas. Excepcionalmente, foram planejadas para colheita 12 árvores referentes a 7 espécies com baixa densidade populacional (atraca, tamburil, gabiroba, estopeiro, tanibuca, cravin, cedrorana), atendendo ao critério de sanidade ou para testes experimentais da madeira (Tabela 9). A conservação dessas espécies pretende favorecer a regeneração natural e ampliar sua população em colheitas futuras. 
Tabela 9 - Número absoluto e relativo de árvores inventariadas e planejadas para colheita de algumas espécies com baixa densidade populacional, no IF $100 \%$, na área de manejo florestal da Fazenda Shet, em Dom Eliseu, Pará

Table 9 - Absolute and relative number of trees inventoried and planned to harvest some species with low population density, in the $100 \%$ IF, in the forest management area of Shet

Farm, in Dom Eliseu, Pará

\begin{tabular}{|c|c|c|c|c|c|c|}
\hline & Nome científico & Nome popular & N. inv. & $\%$ inv. & N./ha & N. colheita \\
\hline 1 & $\begin{array}{l}\text { Quiina } \\
\text { amazonica }\end{array}$ & Quina-rosa & 2 & 0,004 & 0,004 & 0 \\
\hline 2 & Licania spp. & Casca-seca & 3 & 0,007 & 0,006 & 0 \\
\hline 3 & Lecythis pisonis & Sapucaia & 13 & 0,028 & 0,024 & 0 \\
\hline 4 & $\begin{array}{l}\text { Hymenaea } \\
\text { parvifolia }\end{array}$ & Jatobá-curuba & 14 & 0,030 & 0,026 & 0 \\
\hline 5 & Micrandra elata & Seringarana & 9 & 0,020 & 0,028 & 0 \\
\hline 6 & Apuleia leiocarpa & Amarelão & 22 & 0,048 & 0,041 & 0 \\
\hline \multicolumn{3}{|c|}{ Subtotal } & 63 & 00,147 & 00,129 & 0 \\
\hline \multicolumn{3}{|c|}{ Espécies $\leq 0,15$ arv.ha $^{-1}(53 \mathrm{sp})}$. & 927 & 02,015 & 01,731 & 12 \\
\hline \multicolumn{3}{|c|}{ Demais (53 sp.) } & 45.085 & 97,980 & 82,455 & 10.659 \\
\hline \multicolumn{3}{|c|}{ Total geral } & 46.012 & 100,000 & 85,907 & 10.671 \\
\hline
\end{tabular}

Fonte: Autores (2017)

Em que: N. inv. - número de árvores inventariadas; \% inv. - percentual de árvores inventariadas; N. - número de árvores; IF - Inventário Florestal.

\section{Conclusões}

Florestas degradadas, semelhantes às deste estudo, podem ter colheitas a cada 10 e/ou 12 anos, provenientes das árvores com problemas de sanidade, da qualidade do fuste (2 e 3), árvores de plantio em clareiras, da regeneração de espécies mais abundantes, do excedente da distribuição diamétrica, bem como das demais árvores que atingem estado adequado para colheita.

Os critérios para colheita de árvores propostos neste estudo, em floresta natural degradadas na Amazônia, são uma forma de manejo para melhorar a qualidade da floresta, favorecer o aumento das populações de espécies que passaram por intensa exploração no passado, podendo ser alternativa viável economicamente devido ao uso de árvores normalmente não utilizadas e considerando ciclos menores para colheita comparado ao que é previsto na legislação vigente.

\section{Agradecimentos}

Esta pesquisa fez parte do projeto "Rede de inovação da cadeia produtiva florestal madeireira para promoção do desenvolvimento sustentável do Estado do Pará - Rede Biomassa Florestal", também chamado projeto "Sub-Bosque" (2009-2014), financiado pela Fapespa (Fundação de Amparo à Pesquisa) para execução do estudo em parceria entre Embrapa Amazônia Oriental e o Grupo Arboris. 


\section{Referências}

BRAGA, A. J. T.; GRIFFITH, J. J.; PAIVA, H. N. de; MEIRA NETO, J. A. A. Composição do banco de sementes de uma floresta semidecidual secundária considerando o seu potencial de uso para recuperação ambiental. Revista Árvore. v. 32, n. 6, p. 1089-1098, dez. 2008.

BRASIL. Conselho Nacional de Meio Ambiente. CONAMA. Resolução no 406, 2 de fevereiro de 2009. Diário Oficial da União. Brasília, DF, 06 fev. 2009. Seção 1. p. 100.

BRASIL. Ministério do Meio Ambiente. MMA. Instrução Normativa n 5, de 11 de dezembro de 2006. Diário Oficial da República Federativa do Brasil. Brasília, DF, 5 mar. 2006.

BRASIL. Ministério do Meio Ambiente. MMA. Instrução Normativa no 1, de 13 de fevereiro 2015. Diário Oficial da União. Brasília, DF, 13 fev. 2015. n. 31, Seção 1, p. 67.

BRASIL. Ministério do Meio Ambiente. MMA. Lei № 12.651, de 28 de maio de 2012. Diário Oficial da União. Brasília, DF, 28 mai. 2012. p. 1.

BRASIL. Ministério do Meio Ambiente. MMA. Lei № 4.771, de 15 de setembro de 1965. Diário Oficial da República Federativa do Brasil. Brasília, DF, 15 set. 1965. p. 9529.

BRASIL. Ministério do Meio Ambiente. MMA. Lei № 9.605, de 12 de fevereiro de 1998. Diário Oficial da República Federativa do Brasil. Brasília, DF, 12 fev. 1998. p. 1.

FERREIRA, J. N. et al. Degradação florestal na Amazônia: como ultrapassar os limites conceituais, científicos e técnicos para mudar esse cenário. Belém, PA: Embrapa Amazônia Oriental, 2015. 29 p.

FIGUEIREDO FILHO, A.; DIAS, A. N.; STEPKA, T. F.; SAWCZUK, A. R. Crescimento, mortalidade, ingresso e distribuição diamétrica em floresta ombrófila mista floresta. Curitiba, PR, v. 40, n. 4, p. 763-776, out./dez. 2010.

FONTES, C. G. Revelando as causas e a distribuição temporal da mortalidade arbórea em uma floresta de terra-firme na Amazônia Central. 2012. 63f. Dissertação (Mestrado em Ciências de Floresta Tropicais) Instituto Nacional de Pesquisas da Amazônia, Manaus, 2012.

FONTES, C. G.; CHAMBERSB, J. Q.; HIGUCHI, N. Revealing the causes and temporal distribution of tree mortality in Central Amazonia, Forest Ecology and Management, v. 424, p. 177-183, May 2018.

GARZÓN, G.; PAOLA L. Conocimiento tradicional sobre las plantas medicinales de yarumo (Cecropia sciadophylla), carambolo (Averrhoa carambola) y uña de gato (Uncaria tomentosa) en el resguardo indígena de Macedonia, Amazonas. Luna Azul, v. 43, p. 386-414, jul. 2016.

IWAKIRI, S.; ZELLER, F.; PINTO, J. A.; RAMIREZ, M. G. L.; SOUZA, M. M.; SEIXAS, R. Avaliação do potencial de utilização da madeira de Schizolobium amazonicum "Paricá" e Cecropia hololeuca "Embaúba" para produção de painéis aglomerados. Acta Amazonica, v. 40, n. 2, p. 303-308, nov. 2010.

INSTITUTO BRASILEIRO DE GEOGRAFIA ESTATÍSTICA. IBGE. Manual técnico da vegetação brasileira: Sistema fitogeográfico, inventário das formações florestais e campestres, técnicas e manejo de coleções botânicas, procedimentos para mapeamentos. Rio de Janeiro, 2012. 92 p.

INSTITUTO DE DESENVOLVIMENTO ECONÔMICO, SOCIAL E AMBIENTAL DO PARÁ. IDESP. Estatística municipal de Dom Eliseu. Dom Eliseu, Pará, 2014. 47 p.

INSTITUTO DO HOMEM E MEIO AMBIENTE DA AMAZÔNIA. IMAZON. Fatos Florestais da Amazônia. Belém, Pará, 2005. 140 p.

INSTITUTO DO HOMEM E MEIO AMBIENTE DA AMAZÔNIA. IMAZON. Fatos Florestais da Amazônia. Belém, Pará, 2010. 124 p.

LIMA, J. P. C.; LEÃO, J. R. A. Dinâmica de Crescimento e Distribuição Diamétrica de Fragmentos de Florestas Nativa e Plantada na Amazônia Sul Ocidental. Floresta e Ambiente, Rio de Janeiro, v. 20, n. 1, p. 70-79, jan./ 
mar. 2013.

LIOCOURT, F. De. L'aménagement des sapinières. Paris: Société Forestière de Franche-Comté et Belfort, 1898.

FEITOSA, T. R. Caracterização, diversidade e similaridade florística de uma floresta intensivamente explorada no município de Dom Eliseu, sudeste do Estado do Pará. 2015. $41 \mathrm{f}$. Monografia (Trabalho de conclusão do curso de Engenharia Florestal) - Universidade Federal Rural da Amazônia, Belém, 2015.

MEYER, H. A. Structure, growth, and drain in balanced uneven-aged forests. Journal of Forestry, Bethesda, n. 52, v. 2, p. 85-92, 1952.

PARÁ (Estado). Secretaria de Estado e Meio Ambiente. SEMA. Gerência de Sistemas de Comercialização e Transporte de Produtos Florestais. Autorização para Exploração Florestal no 2077/2012 (AUTEF). Belém, 2013. Disponível em: http://monitoramento.sema.pa.gov.br/simlam/ index.htm. Acesso em: 15 dezembro de 2016.

PARÁ (Estado). Secretaria de Estado e Meio Ambiente. SEMA. Gerência de Sistemas de Comercialização e Transporte de Produtos Florestais. Autorização para Exploração Florestal no 2377/2013 (AUTEF). Belém, 2014. Disponível em: http://monitoramento.sema.pa.gov.br/simlam/ index.htm. Acesso em: 15 dezembro de 2016.

PEREIRA, P. C. G. Potencial silvicultural das espécies do gênero Cecropia na Amazônia. 2015. 45 f. Dissertação (Mestrado em Ciências Florestais) - Universidade Federal Rural da Amazônia, Belém, 2015.

POGGIANI, F. Florestas para fins energéticos e ciclagem de nutrientes. Série Técnica IPEF. 1 (2):D1D11. 1980.

SABOGAL, C. et al. Diretrizes técnicas de manejo para produção madeireira mecanizada em florestas de terra firme na Amazônia brasileira. Belém: Embrapa Amazônia Oriental, 2009. 217p.

SALES, A. et al. Estimation of the commercial height of trees with laser meter: a viable alternative for forest management in the Brazilian Amazon. Ecology and Evolution. p. 1-6, 2018.

SCHWARTZ, G.; PEREIRA, P. C. G.; SIVIERO, M. A.; PEREIRA, J. F.; RUSCHEL, A. R.; YARED, J. A. G. Enrichment planting in logging gaps with Schizolobium parahyba var. amazonicum (Huber ex Ducke) Barneby: A financially profitable alternative for degraded tropical forests in the Amazon. Forest Ecology and Management. 390:166-172, 2017.

SIVIERO, M. A. et al. Nossa indústria madeireira rumo à sustentabilidade. Referência Florestal, Curitiba, p. 72-73, dez. 2009. Artigo técnico.

SIVIERO, M. A.; LOURENÇO, R. F.; PACHECO, A. Quebrando paradigmas. Referência Florestal, Curitiba, p. 45-47, fev. 2007. Informe técnico.

SIVIERO, M. A. et al. É possível inovar no manejo de florestas naturais? Referência Florestal, Curitiba, p. 94-96, jul. 2011. Artigo técnico.

SOUZA, D. R.; SOUZA, A. L. Emprego do método BDq de seleção após a exploração florestal em floresta ombrófila densa de terra firme, Amazônia Oriental. Revista Árvore, Viçosa, v. 29, n. 4, p. 617-625, jul./ago. 2005.

VATRAZ, S. et al. Efeitos de tratamentos silviculturais sobre o crescimento de Laetia procera (Poepp.) Eichler em Paragominas, PA, Brasil. Scientia Forestalis, Piracicaba, v. 40, n. 93, p. 095-102, mar. 2012.

VATRAZ, S. et al. Efeito da exploração de impacto reduzido na dinâmica do crescimento de uma floresta natural. Scientia Forestalis, Piracicaba, v. 44, n. 109, p. 261-271, mar. 2016.

VIEIRA, D. S. et al. Comparação estrutural entre floresta manejada e não manejada na comunidade Santo Antônio, Estado do Pará. Ciência Florestal, Santa Maria, v. 24, n. 4, p. 1067-1074, out./dez. 2014. 